\title{
Modified versus conventional ultrafiltration in pediatric cardiac surgery: A meta-analysis of randomized controlled trials comparing clinical outcome parameters
}

\author{
Norifumi Kuratani, MD, PhD, ${ }^{a}$ Piyaporn Bunsangjaroen, MD, ${ }^{\mathrm{b}}$ Thanaphon Srimueang, MD, \\ Eiji Masaki, MD, PhD, ${ }^{\mathrm{c}}$ Takaaki Suzuki, $\mathrm{MD}, \mathrm{PhD},{ }^{\mathrm{d}}$ and Toshiyuki Katogi, $\mathrm{MD}, \mathrm{PhD}^{\mathrm{d}}$
}

Objective: Although previous studies have demonstrated that modified ultrafiltration improves laboratory parameters in pediatric cardiac surgery, the clinical outcome data have been inconsistent. We performed a meta-analysis of randomized controlled trials comparing modified versus conventional ultrafiltration.

\begin{abstract}
Methods: We conducted a comprehensive search of the literature to identify clinical trials that met our inclusion criteria. To be included, studies had to be prospective randomized trials that compared modified ultrafiltration and conventional ultrafiltration in pediatric cardiac surgery using cardiopulmonary bypass. We focused on the following outcome variables: hematocrit and mean arterial blood pressure after cardiopulmonary bypass, amount of chest tube drainage after surgery, time to extubation, and length of stay in the intensive care unit. The random effects model was used to determine the pooled effect estimates. The estimators of treatment effects were expressed as the weighted mean difference with $95 \%$ confidence intervals. The heterogeneity of collected data was also evaluated.
\end{abstract}

Results: We screened 54 studies, 8 of which satisfied our inclusion criteria. Combined analysis revealed that modified ultrafiltration resulted in significantly higher postbypass hematocrit and higher mean arterial blood pressure. Benefits in postoperative blood loss, ventilator time, and intensive care unit stay were not apparent. There was significant heterogeneity among the studies surveyed.

Conclusions: The advantage of modified ultrafiltration over conventional ultrafiltration consists of significant improvement of clinical conditions in the immediate postbypass period. The postoperative outcome parameters were not significantly influenced. We should also take into account possible clinical or methodologic variations in the currently available ultrafiltration studies. (J Thorac Cardiovasc Surg 2011;142:861-7)

Earn CME credits at

http://cme.ctsnetjournals.org

Although cardiopulmonary bypass (CPB) is essential in pediatric cardiac surgery, it is widely known that CPB itself can contribute to the development of significant morbidity after surgery. Children, especially those who are small, he-

\footnotetext{
From the Division of Pediatric Anesthesia, ${ }^{a}$ International University of Health and Welfare Hospital, Tochigi, Japan; Department of Anesthesiology, ${ }^{\text {b }}$ Khon Khan University, Khon Khan, Thailand; Division of Dento-oral Anesthesiology, ${ }^{\mathrm{c}}$ Tohoku University Graduate School of Dentistry, Miyagi, Japan; and Department of Pediatric Cardiac Surgery, ${ }^{\mathrm{d}}$ Saimata International Medical Center, Saitama, Japan.

This work was funded solely from the departmental resources of the International University of Health and Welfare and Saitama Medical University.

Disclosures: Authors have nothing to disclose with regard to commercial support.

Received for publication Aug 3, 2010; revisions received Feb 16, 2011; accepted for publication April 4, 2011; available ahead of print May 6, 2011.

Address for reprints: Norifumi Kuratani, MD, PhD, Division of Pediatric Anesthesia, International University of Health and Welfare Hospital, 537-3 Iguchi, Nasushio-

bara, Tochigi 329-2763 Japan, Tel: +81-287-37-2221, Fax: +81-287-39-3001

(E-mail: nori-kuratani@umin.ac.jp).

$0022-5223 / \$ 36.00$

Copyright (c) 2011 by The American Association for Thoracic Surgery

doi:10.1016/j.jtcvs.2011.04.001
}

modiluted, and cooled or those who have experienced lengthy CPB times, are at increased risk for the development of many of the complications associated with CPB. Because hemodilution by CPB priming is significant in pediatric patients, ultrafiltration to remove excess water can counteract the deleterious effects of CPB. Because conventional ultrafiltration (CUF), which is carried out while $\mathrm{CPB}$ is running, offers limited filtration efficiency, modified ultrafiltration (MUF) was introduced ${ }^{1}$ and has gained popularity over the past 2 decades. Because MUF is performed immediately after the termination of CPB, MUF removes excess fluid with greater efficiency than CUF. The expected benefits of MUF include a reduction of total body water and the removal of inflammatory mediators, both of which contribute to restoring normal organ function and improving outcome.

The potential benefits of MUF have been supported by numerous laboratory and clinical studies. Although MUF is decidedly beneficial in terms of certain laboratory parameters, the available data on clinical outcomes are insufficient and inconsistent. It is worth considering that MUF has some potential disadvantages, including possible technical complications. The question has therefore been raised as to whether MUF should be performed on all pediatric patients 


\section{Abbreviations and Acronyms \\ $\mathrm{CPB}=$ cardiopulmonary bypass \\ $\mathrm{CI}=$ confidence interval \\ CUF $=$ conventional ultrafiltration \\ ICU = intensive care unit \\ MUF $=$ modified ultrafiltration \\ $\mathrm{WMD}=$ weighted mean difference}

who have undergone CPB. The MUF circuit has an artificial surface that can elicit additional inflammatory responses. MUF requires additional time (typically, 15-20 minutes) after the termination of $\mathrm{CPB}$, and it incurs additional costs. To support the rational application of MUF to pediatric cardiac patients, we need unbiased data regarding its clinical parameters and outcomes.

Meta-analysis is a statistical tool that can be used to evaluate published data in both qualitative and quantitative ways, accounting for variations in characteristics that can influence the overall estimate of outcomes of interest. The statistical aggregation of randomized trials through metaanalysis allows for increased statistical power in detecting potential differences in clinical outcomes. In this report, we present a meta-analysis of randomized controlled trials intended to clarify the clinical benefit of MUF in pediatric cardiac surgery.

\section{METHODS}

We conducted a systematic review according to the Quality of Reporting of Meta-analyses recommendations developed to improve the quality of meta-analyses. $^{2}$

A comprehensive search of the literature was performed using MEDLINE, the American College of Physicians Journal Club database, the Cochrane Database of Systematic Reviews, the Cochrane Central Register of Controlled Trials, and the Database of Abstracts of Reviews of Effects. The following text searches and search headings were used individually and in combination: "modified ultrafiltration," "cardiac surgery," "child," "infant," and "cardiopulmonary bypass." A manual search of references listed in reports and reviews was also performed. Only articles written in English were included. The date of the most recent search was May 23, 2010. Every effort was made to find studies that reported the clinical outcome data comparing MUF versus CUF in pediatric cardiac surgery using $\mathrm{CPB}$. To be included in our analysis, studies had to be prospective randomized trials comparing MUF and CUF in pediatric cardiac surgery using CPB. Studies that compared MUF and control patients without any ultrafiltration technique were not included, because the benefits of ultrafiltration in pediatric CPB are widely acknowledged and CPB management without any ultrafiltration would not reflect actual clinical practice. Two authors (P.B., T.S.) independently assessed each article to ensure that it met the aforementioned inclusion criteria. Disagreements were resolved by consensus and the final decision was made by the referee author (N.K.).

Unmasked quality assessments on the selected published studies were carried out by 2 investigators (P.B., T.S.) on composite aspects of study quality ( 5 aspects in total, each scored as 0 or 1: randomization, comparability, standardized CPB management protocol, standardized postoperative care, withdrawals). Differences in opinion were settled by consensus and the final decision was made by the referee author (N.K.). Data abstraction was also performed independently by 2 authors (P.B., T.S.) using standardized data collection forms. Inasmuch as the proposed clinical advantages of MUF include hemoconcentration, reduced blood loss, and improvement of cardiovascular and respiratory function, ${ }^{3}$ we focused on the following outcome variables: hematocrit and blood pressure after $\mathrm{CPB}$, amount of chest tube drainage within 48 hours after surgery, time to extubation, and length of stay in the intensive care unit (ICU). Morbidities attributed to MUF and mortalities as a result of any cause were also collected. The clinical data, expressed as mean \pm standard deviation, were extracted from each article. When the standard error was reported, we determined the standard deviation as the standard error multiplied by the square root of the number of subjects. Variables that were not reported numerically were estimated by extrapolating data from the published figures. When the median data were reported, the mean and standard deviation were estimated by assuming that the mean was equivalent to the median and that the standard deviation was half of the median value.

All statistical analyses were performed using RevMan 4.2.10 (The Cochrane Collaboration, Oxford, United Kingdom). The random effects model was used to determine the pooled effect estimates. The estimators of treatment effects were expressed as the weighted mean difference (WMD) with $95 \%$ confidence intervals (CIs). Because eligible studies showed clinical and methodologic diversity, the heterogeneity of collected data was assessed using a homogeneity test based on the $\chi^{2}$ test and $\mathrm{I}^{2}$. The $\mathrm{I}^{2}$ statistic was used to assess the impact of heterogeneity on the results. ${ }^{4}$ This statistic indicates the percentage of variability in effect estimates that is due to heterogeneity rather than sampling error. ${ }^{4}$ Owing to the low power of this test, especially when trials have a small sample size or are few in number, we determined a minimum cutoff $P$ value of .10 and $\mathrm{I}^{2}$ value of $50 \%$ as a threshold of homogeneity to avoid false negative results; $P<.10$ and $\mathrm{I}^{2}>50 \%$ indicated heterogeneity and the combined results should therefore be interpreted with caution.

\section{RESULTS}

\section{Screening Process and Study Selection}

Using electronic databases and manual search, we initially identified 54 articles for review. Of those, 14 studies were excluded in the primary screening inasmuch as they were unrelated studies or review articles and 1 was a case series report. The other 40 articles were thoroughly checked to ensure that they met our inclusion criteria and 25 studies were excluded because they did not. The clinical outcome data that we focused on were not available in 6 studies. One published article that was retracted later by the authors was excluded from the final analysis. Thus, 8 studies $^{5-12}$ were identified through our defined search strategy that fulfilled the inclusion criteria in that they contained the necessary data for the planned comparison. The process of identifying eligible studies is illustrated in Figure 1.

\section{Description of Studies}

The details of selected trials are summarized in Table 1. In total, 438 patients were studied, including 232 CUF patients and 206 MUF patients. In 5 studies, ${ }^{5,6,8,10,12}$ MUF group patients also underwent CUF or diluted ultrafiltration during rewarming periods of CPB. Three studies ${ }^{5,6,12}$ concluded that MUF offered favorable clinical outcomes whereas the other 5 studies $^{7-11}$ reported no clinically significant difference between MUF and CUF. Of the 8 studies, 


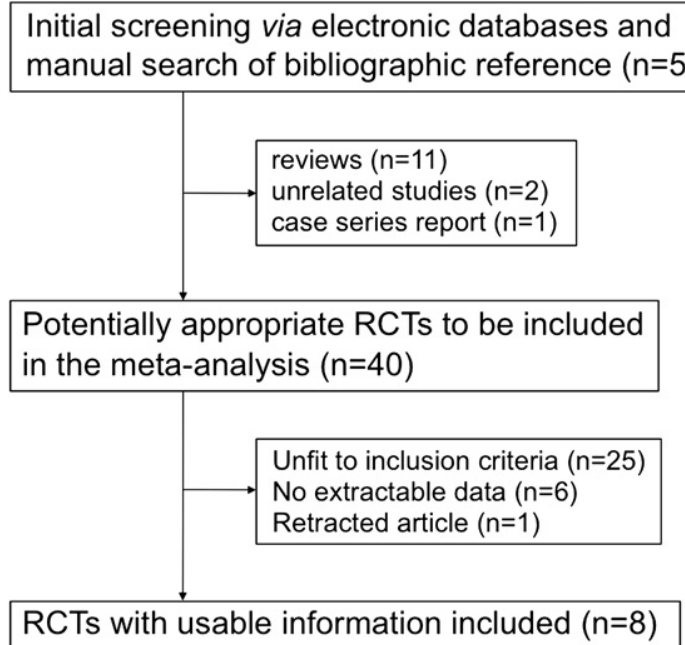

FIGURE 1. Meta-analysis flow chart. $R C T$, Randomized controlled trial.

1 study ${ }^{5}$ used venovenous MUF and the others used arteriovenous MUF. ${ }^{6-12}$ In 3 studies, ${ }^{9-11}$ the fluid volume of ultrafiltration was described in the study protocol. The amount of ultrafiltrate was reported in 6 studies, ${ }^{5,7,9-12}$ and 4 studies $^{5,7,10,12}$ reported that greater amounts of filtrate were obtained in MUF group patients. The median study quality of the selected trials was 3.5 (range, 2-5). Williams and associates $^{8}$ compared CUF and MUF with and without CUF. The clinical outcome data of MUF with CUF were adopted for our analysis. In the study by Berdat and colleagues, ${ }^{11}$ 2 different brands of ultrafilters were used. Inasmuch as they failed to find any significant differences between the
2 different ultrafilters, we combined the outcome data of the 2 ultrafilters together for the meta-analysis.

\section{Mortality, Morbidity, and Technical Complications}

Only 1 study $^{8}$ reported technical problems related to MUF. Williams and coworkers ${ }^{8}$ reported 2 cases of early termination of MUF owing to significant hypotension. Bando and colleagues ${ }^{5}$ declared no complications related to MUF in their study. Other studies did not mention the technical issues related to MUF. Four studies ${ }^{5,7,8,11)}$ reported the overall mortality and morbidity of study patients. Bando and associates ${ }^{5}$ reported 1 postoperative death in the MUF group. The patient died of low cardiac output after an arterial switch repair that was followed by 5 days of extracorporeal circulatory support. Wang and coworkers ${ }^{7}$ reported that 1 patient in the MUF group and 1 in the CUF group died of cardiac failure and could not be weaned from CPB. Two late deaths in the MUF group were reported in the study by Williams and associates. ${ }^{8}$ Berdat and colleagues ${ }^{11}$ reported 1 death in the CUF group and several complications in both groups. In the studies we selected for analysis, no mortalities attributed to MUF were reported.

\section{Combined Analysis}

Postbypass hematocrit (Figure 2, A). The hematocrit data for the postbypass period were reported in 5 studies, ${ }^{5-7,9,12}$ which included a total of 317 patients. All studies, except that reported by Wang's group, ${ }^{7}$ reported the hematocrit value immediately after the termination of $\mathrm{CPB}$ or MUF. Wang and associates ${ }^{7}$ reported the hematocrit value

TABLE 1. Summary of the studies included in the meta-anlaysis

\begin{tabular}{|c|c|c|c|c|c|}
\hline Study & $\begin{array}{l}\text { Ultrafiltration } \\
\text { (no. of patients) }\end{array}$ & $\begin{array}{l}\text { Patient age* } \\
\quad(\text { mo })\end{array}$ & $\begin{array}{l}\text { Study } \\
\text { quality }\end{array}$ & Technical complication & Remarks \\
\hline \multirow[t]{2}{*}{ Bando et $\mathrm{al}^{5}$} & CUF $(\mathrm{N}=50)$ & $30.1 \pm 42.2$ & 5 & No MUF-related complication & $\begin{array}{l}\text { "Complex" congenital heart } \\
\text { surgery only. }\end{array}$ \\
\hline & $\mathrm{DUF}+(\mathrm{v}-\mathrm{v}) \mathrm{MUF}(\mathrm{N}=50)$ & $17.7 \pm 20.7$ & & & One death in MUF group \\
\hline \multirow[t]{2}{*}{ Server et $\mathrm{al}^{6}$} & $\operatorname{CUF}(\mathrm{N}=14)$ & $12.94 \pm 12.98$ & 2 & Not reported & \\
\hline & $\mathrm{CUF}+(\mathrm{a}-\mathrm{v}) \mathrm{MUF}(\mathrm{N}=13)$ & $9.38 \pm 1.94$ & & & \\
\hline Wang et $\mathrm{al}^{7}$ & $\begin{array}{l}\text { CUF }(N=26) \\
(a-v) \operatorname{MUF}(N=24)\end{array}$ & $\begin{aligned} 43.6 & \pm 33 \\
62.16 & \pm 46.44\end{aligned}$ & 3 & Not reported & $\begin{array}{l}\text { One patient in each group died of } \\
\text { cardiac failure. }\end{array}$ \\
\hline William et $\mathrm{al}^{8}$ & $\begin{array}{l}\text { CUF }(N=19) \\
\text { CUF }+(a-v) \operatorname{MUF}(N=21)\end{array}$ & $\begin{array}{l}2.0 \pm 2.2 \\
2.9 \pm 3.45\end{array}$ & 5 & $\begin{array}{l}\text { Two cases, MUF terminated early } \\
\text { because of hypotension }\end{array}$ & Two late deaths in MUF group \\
\hline Thompson et $\mathrm{al}^{9}$ & $\begin{array}{l}\mathrm{CUF} \dagger(\mathrm{N}=67) \\
(\mathrm{a}-\mathrm{v}) \mathrm{MUF} \dagger(\mathrm{N}=43)\end{array}$ & $\begin{aligned} 9.0 & \pm 11.3 \\
12.6 & \pm 14.1\end{aligned}$ & 2 & Not reported & \\
\hline Mahmoud et al ${ }^{10}$ & $\begin{array}{l}\operatorname{CUF}(N=20) \\
\mathrm{CUF}+(\mathrm{a}-\mathrm{v}) \mathrm{MUF} \dagger(\mathrm{N}=20)\end{array}$ & $\begin{array}{l}11.8 \pm 3.3 \\
13.1 \pm 4.1\end{array}$ & 3 & Not reported & \\
\hline \multirow[t]{2}{*}{ Berdat et $\mathrm{al}^{11}$} & $\mathrm{CUF} \dagger(\mathrm{N}=21)$ & $23.4 \pm 16.2$ & 5 & Not reported & $\begin{array}{c}\text { The data of } 2 \text { types of ultrafilters } \\
\text { were combined for analysis. }\end{array}$ \\
\hline & $(\mathrm{a}-\mathrm{v}) \mathrm{MUF} \dagger(\mathrm{N}=20)$ & $17.3 \pm 16.6$ & & & $\begin{array}{l}\text { One death in CUF, other } \\
\text { complications in each group }\end{array}$ \\
\hline Aggarwal et al ${ }^{12}$ & $\begin{array}{l}\operatorname{CUF}(\mathrm{N}=15) \\
\mathrm{CUF}+(\mathrm{a}-\mathrm{v}) \mathrm{MUF} \dagger(\mathrm{N}=15)\end{array}$ & $\begin{aligned} 33.6 & \pm 13.9 \\
30 & \pm 20.8\end{aligned}$ & 4 & Not reported & \\
\hline
\end{tabular}

$C U F$, Conventional ultrafiltration; $D U F$, dilutional ultrafiltration; $M U F$, modified ultrafiltration; $v-v$, venovenous; $a-v$, arteriovenous. *Values are given as mean \pm standard deviation. $†$ The filtration volume was standardized. 


\section{Post-bypass hematocrit (\%)}

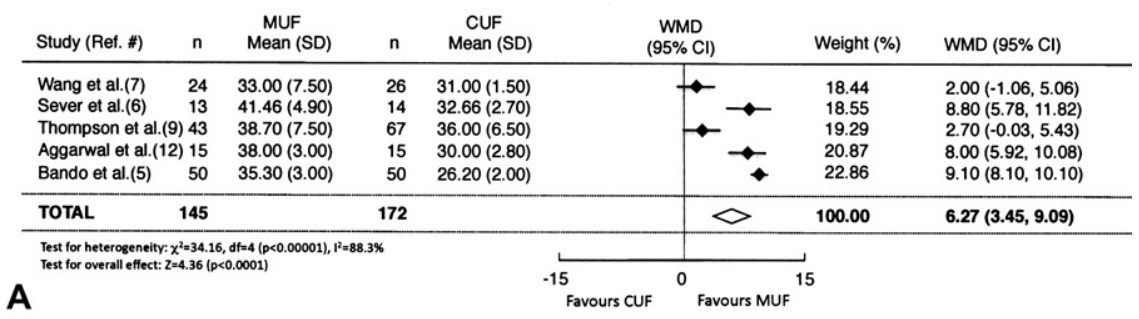

Post-bypass mean arterial pressure $(\mathrm{mmHg})$

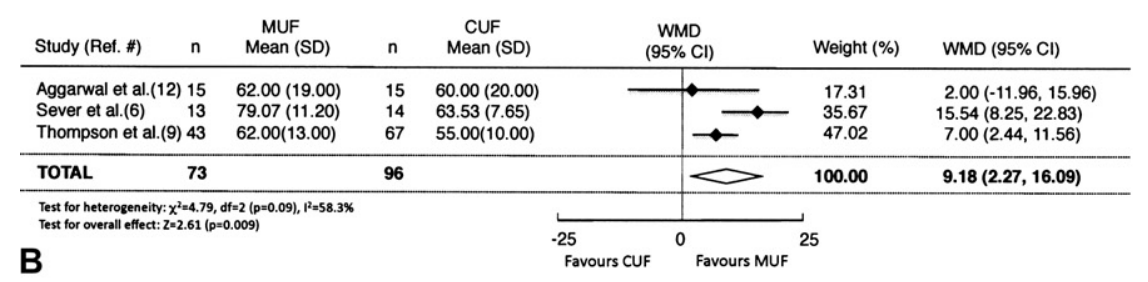

Chest tube drainage $(\mathrm{ml} / \mathrm{kg})$

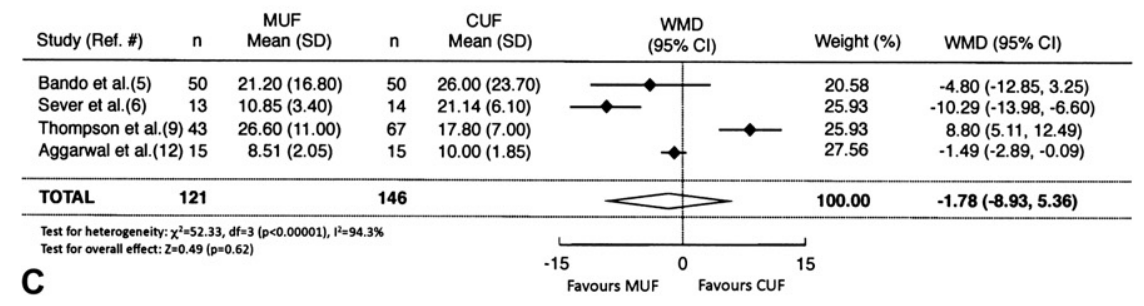

Post-operative ventilation time (hour)

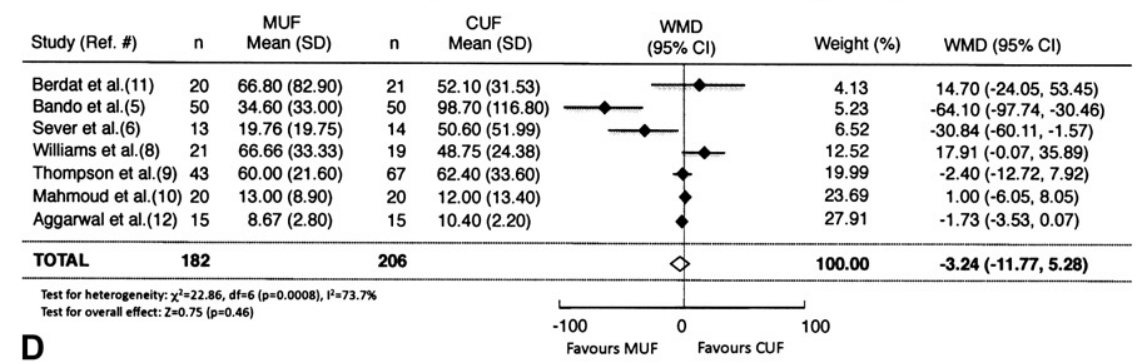

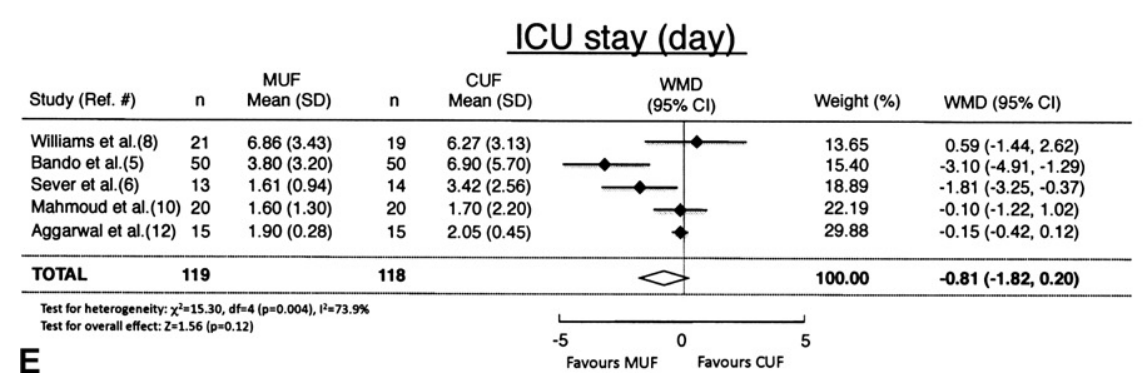

FIGURE 2. Meta-analysis of clinical outcome parameters in MUF compared with CUF. Effect sizes of MUF are represented by the weight mean difference, shown as black diamonds. Horizontal lines represent the lower and upper limits of the $95 \%$ confidence intervals. The open diamond indicates the pooled result. $M U F$, Modified ultrafiltration; $C U F$, conventional ultrafiltration; WMD, weighted mean difference; $C I$, confidence interval; Ref, reference.

at the end of the operation. We treated these values as postbypass hematocrit data. Combined analysis revealed that MUF resulted in significantly higher postbypass hematocrit levels $(\mathrm{WMD}=6.27$; 95\% CI, 3.45-9.09; $P<.0001)$. Sig- nificant heterogeneity was also revealed $\left(\chi^{2}=34.16 ; d f=4\right.$ $\left.[P<.00001] ; \mathrm{I}^{2}=88.3 \%\right)$.

Postbypass mean arterial blood pressure (Figure 2, $\boldsymbol{B})$. The arterial blood pressure data in the postbypass 
period were available in 3 studies. ${ }^{6,9,12}$ Aggarwal and associates $^{12}$ reported the systolic and diastolic pressure data and we calculated MAP using the following equation:

$$
\begin{aligned}
\text { MAP }= & \text { diastolic pressure }+1 / 3 \\
& (\text { systolic pressure }- \text { diastolic pressure })
\end{aligned}
$$

The pooled results showed a significant improvement in systemic blood pressure favoring the MUF group, with a WMD of 9.18 (95\% CI, 2.27-16.09; $P=.009)$. The heterogeneity was statistically significant $\left(\chi^{2}=4.79 ; d f=2\right.$ $\left.[P=.09] ; \mathrm{I}^{2}=58.3 \%\right)$.

Chest tube drainage (Figure 2, $C$ ). The mean amount of chest tube drainage was reported in 4 trials. ${ }^{5,6,9,12}$ Bando, ${ }^{5}$ Sever, ${ }^{6}$ and their associates reported the amount of blood loss during the first 24 hours only. Because the amount of chest tube output on the second postoperative day is usually small, we considered these data sufficient for inclusion in our analysis. If an article reported the amount of chest tube drainage in milliliters, the data were converted to milliliter per kilogram using the mean body weight data reported. Of the 4 studies reporting chest tube drainage data, $3^{5,6,12}$ ( 2 of which reported statistically significant results ${ }^{6,12}$ ) concluded that decreasing blood loss through the chest tube had a favorable effect on the overall outcome. Thompson and coworkers ${ }^{9}$ reported significantly increased blood loss in the MUF group but did not discuss any potential reasons for it. The pooled analysis of these 4 studies failed to identify a statistically significant difference between MUF and the control group (WMD $=-1.78 ; 95 \% \mathrm{CI},-8.93$ to 5.36 ; $P=.62)$. Statistical heterogeneity was found between trials $\left(\chi^{2}=52.33 ; d f=3[P<.00001] ; \mathrm{I}^{2}=94.3 \%\right)$.

Duration of mechanical ventilation (Figure 2, D). All studies, ${ }^{5,6,8-12}$ except that reported by Wang and associates, ${ }^{7}$ reported the duration of mechanical ventilation after surgery. The postoperative ventilatory management and extubation criteria were mentioned in $4^{5,8,10,12}$ of 7 studies. Two articles ${ }^{5,6}$ reported that MUF significantly shortened the postoperative ventilatory time compared with CUF. Other studies reported no significant difference between CUF and MUF patients. Combined analysis indicated that there was no difference between MUF and the control group in terms of ventilation time (WMD $=-3.24 ; 95 \%$ $\mathrm{CI},-11.77$ to $5.28 ; P=.46)$. We found significant heterogeneity among trials $\left(\chi^{2}=22.86 ; d f=6[P=.0008]\right.$; $\left.\mathrm{I}^{2}=73.7 \%\right)$.

Duration of ICU stay (Figure 2, E). Data regarding the length of ICU stay were available for $5^{5,6,8,10,12}$ of the 8 trials included in this meta-analysis. No studies described the ICU discharge criteria. The combined results failed to show that MUF shortened the ICU stay on average (WMD $=-0.81$; $95 \%$ CI, -1.82 to $0.20 ; P=0.12$; heterogeneity: $\left.\chi^{2}=15.30 ; d f=4[P=.004] ; \mathrm{I}^{2}=73.9 \%\right)$.

\section{DISCUSSION}

In this meta-analysis, evidence from currently available randomized controlled studies regarding ultrafiltration in pediatric cardiac surgery revealed that MUF augmented hemoconcentration and facilitated the restoration of circulation, as compared with CUF. However, postoperative outcome parameters, including chest tube drainage, ventilator time, and ICU stay, were not significantly influenced by MUF. These findings suggest that MUF could contribute to improving the clinical conditions immediately after $\mathrm{CPB}$, although its impact on the overall clinical outcome might not be significant.

Hematocrit levels after the termination of CPB were significantly higher in MUF patients than in CUF patients. The higher hematocrit in MUF patients reflects the higher efficiency of hemoconcentration in MUF compared with CUF. High hematocrit levels after bypass can help reduce the need for transfused blood and thereby offer the significant benefit of minimizing homologous blood exposure. Of the 8 studies we identified in this meta-analysis, 2 studies $^{5,6}$ showed a reduction in blood transfusion in MUF patients compared with those who had CUF only.

Our analysis demonstrated that MUF patients showed higher systemic blood pressure after CPB. This higher systemic blood pressure reflects the augmented recovery of the circulatory system in MUF patients. Hypothermic CPB with cardiac arrest is an extremely unphysiologic condition for the circulatory system. Myocardial edema resulting from hemodilution and increased vascular permeability contributes to myocardial dysfunction after CPB. In the dysfunctional heart, myocardial thickness and decreased systolic function are often observed by ultrasound examination in the postbypass period. ${ }^{13}$ Previous studies have illustrated that MUF reduces the edema of the myocardium and facilitates the restoration of normal myocardial function. ${ }^{13,14}$ Another possible cause of higher blood pressure after MUF could be decreased concentrations of anesthetics owing to the filtration process. Hodges and colleagues ${ }^{15}$ measured plasma anesthetic concentration after MUF and showed that the plasma concentration of fentanyl remained stable throughout ultrafiltration. They concluded that the higher blood pressure in the MUF group was not likely a result of the decreased plasma anesthetic level. ${ }^{15}$

In our analysis, MUF failed to decrease the amount of chest tube drainage in the ICU. Coagulopathy and hemostatic difficulty are common after CPB in pediatric patients. Because the coagulation system of a neonate undergoing $\mathrm{CPB}$ is known to be profoundly and globally affected by hemodilution, ${ }^{16} \mathrm{MUF}$ is expected to reverse the adverse effects of hemodilution on the coagulation system. Indeed, previous reports have suggested that MUF increased the concentration of coagulation factors and that it attenuated the coagulopathy associated with CPB. ${ }^{17,18}$ Hemostatic difficulty after CPB 
does not have a simple pathologic cause, however; multiple factors are involved. ${ }^{19}$ Increased inflammatory responses, platelet dysfunction, and increased fibrinolysis are other major factors that should be considered as reasons for abnormal hemostasis. The effects of MUF on preserving platelet function and fibrinolysis have not yet been fully clarified.

Pulmonary dysfunction after CPB is common in pediatric cardiac surgery and may result in significant morbidity and mortality. The reasons for CPB-induced lung injury include increased interstitial lung water owing to hemodilution, lung ischemia during aortic crossclamping, and inflammatory reaction elicited by CPB. Because MUF can eliminate excess water and can ameliorate inflammatory reactions, the advantages of MUF in terms of lung function have been noted and are widely accepted. However, our metaanalysis failed to show the benefit of MUF on postoperative ventilation time. As Mahmoud and associates ${ }^{10}$ have pointed out, the advantages of MUF on lung function might be of limited duration only rather than sustained for long postoperative periods. An alternative view is that the postoperative ventilation time may not reflect the real benefit of MUF in terms of lung function. If we consider the results of previous studies ${ }^{20,21}$ that demonstrated the improvement of various pulmonary parameters, including lung compliances and respiratory indexes, we cannot eliminate the possibility that MUF facilitates the restoration of lung function in the immediate postbypass period.

To counteract pathologic fluid accumulation during CPB, ultrafiltration to remove excess water is now a widely accepted practice in pediatric cardiac surgery. Theoretically, MUF has a much higher efficiency in terms of fluid removal than does CUF, because it is carried out after the termination of CPB. Indeed, previous reports ${ }^{10,12}$ have indicated that the ultrafiltrated fluid volume was larger in MUF. Meanwhile, Thompson and associates ${ }^{9}$ conducted a prospective randomized study to assess the hypothesis that MUF and CUF have similar clinical effects when a standardized volume of fluid is removed. They concluded that hematocrit, hemodynamics, ventricular function, blood product requirements, and postoperative resources used do not differ between pediatric patients receiving CUF and those receiving MUF. ${ }^{9}$ It remains unknown whether the benefits of MUF depend solely on its greater efficiency at fluid removal.

Another potential advantage of ultrafiltration is cytokine removal and inflammatory response attenuation. Surgical trauma and CPB are associated with the production of various kinds of cytokines and inflammatory responses. These effects are most pronounced in pediatric patients. Such inflammatory responses can play a role in eliciting morbidity and mortality in postoperative periods. Indeed, Allan and colleagues $^{22}$ have demonstrated that postoperative interleukin 6 and interleukin 8 are correlated with the length of the ICU stay in infant cardiac surgery. Some studies have reported reduced cytokine levels and reduced inflammatory responses after ultrafiltration. ${ }^{23,24}$ Inasmuch as MUF has higher efficiency in terms of fluid removal, it may be capable of filtering out inflammatory mediators more efficiently as well. However, it is still unknown whether reduction of cytokine levels by ultrafiltration can contribute to favorable outcome in pediatric cardiac surgery. Further study will be necessary to clarify the attenuation of the inflammatory response by MUF and to determine the clinical benefits.

It is important to note some of the limitations of metaanalysis. Each study has different study protocols; this may be the reason for the significant heterogeneity revealed by the $\mathrm{I}^{2}$ test in our meta-analysis. The justification of combining the results of different protocols in the calculation of the WMD and in drawing conclusions is debatable. Factors that may influence study results include the type of ultrafiltration during CPB, type of MUF, duration of ultrafiltration during $\mathrm{CPB}$, volume of ultrafiltrate obtained, end point chosen for termination of MUF, type of hemofilter, concomitant anti-inflammatory therapies, patient characteristics, CPB variables, and complexity of cardiac surgery. In addition, because the meta-analysis is based on published articles, there is a possibility of publication bias. In this study, the omission of the unpublished, nonindexed, or non-English articles that were not included may affect our conclusions. Although we limited our analysis to the literature in English, the effect of excluding non-English trials on the results of a meta-analysis is equivocal. Some data suggest that the exclusion of trials not published in English may actually result in a more conservative estimate of the treatment effect. ${ }^{25}$ This may be related in part to the presence of publication bias where only positive findings are published; this occurs primarily in English-language journals.

In conclusion, meta-analysis of the currently available randomized controlled trials that examined the clinical benefits of MUF over CUF in pediatric cardiac surgery indicates that MUF resulted in significantly higher postbypass hematocrit levels and higher mean arterial blood pressure. Our analysis failed to show a positive impact of MUF in postoperative clinical parameters, including postoperative blood loss, ventilator time, and ICU time. These findings suggest that MUF, compared with CUF, can improve clinical conditions in the immediate postbypass period, although the benefit of MUF on patient overall outcome might not be significant. We must, however, take into account the possible clinical or methodologic variations in the currently available evidence related to MUF.

We thank Ryuichiro Araki, PhD (Community Health Science Center, Saitama Medical University, Saitama, Japan) for his statistical assistance.

\section{References}

1. Naik SK, Knight A, Elliott M. A prospective randomized study of a modified technique of ultrafiltration during pediatric open-heart surgery. Circulation. 1991;84(5 Suppl):III422-31. 
2. Moher D, Cook DJ, Eastwood S, Olkin I, Rennie D, Stroup DF. Improving the quality of reports of meta-analyses of randomized controlled trials: The QUOROM statement. Quality of Reporting of Meta-analyses. Lancet. 1999;354:1896-900.

3. Gaynor JW. The effect of modified ultrafiltration on the postoperative course in patients with congenital heart disease. Semin Thorac Cardiovasc Surg Pediatr Card Surg Annu. 2003;6:128-39.

4. Higgins JP, Thompson SG, Deeks JJ, Altman DG. Measuring inconsistency in meta-analyses. BMJ. 2003;327:557-60.

5. Bando K, Turrentine MW, Vijay P, Sharp TG, Sekine Y, Lalone BJ, et al. Effect of modified ultrafiltration in high-risk patients undergoing operations for congenital heart disease. Ann Thorac Surg. 1998;66:821-7.

6. Sever K, Tansel T, Basaran M, Kafali E, Ugurlucan M, Ali Sayin O, et al. The benefits of continuous ultrafiltration in pediatric cardiac surgery. Scand Cardiovasc J. 2004;38:307-11.

7. Wang MJ, Chiu IS, Hsu CM, Wang CM, Lin PL, Chang CI, et al. Efficacy of ultrafiltration in removing inflammatory mediators during pediatric cardiac operations. Ann Thorac Surg. 1996;61:651-6.

8. Williams GD, Ramamoorthy C, Chu L, Hammer GB, Kamra K, Boltz MG, et al. Modified and conventional ultrafiltration during pediatric cardiac surgery: clinical outcomes compared. J Thorac Cardiovasc Surg. 2006;132:1291-8.

9. Thompson LD, McElhinney DB, Findlay P, Miller-Hance W, Chen MJ, Minami $\mathrm{M}$, et al. A prospective randomized study comparing volumestandardized modified and conventional ultrafiltration in pediatric cardiac surgery. J Thorac Cardiovasc Surg. 2001;122:220-8.

10. Mahmoud AB, Burhani MS, Hannef AA, Jamjoom AA, Al-Githmi IS, Baslaim GM. Effect of modified ultrafiltration on pulmonary function after cardiopulmonary bypass. Chest. 2005;128:3447-53.

11. Berdat PA, Eichenberger E, Ebell J, Pfammatter JP, Pavlovic M, Zobrist C, et al. Elimination of proinflammatory cytokines in pediatric cardiac surgery: analysis of ultrafiltration method and filter type. J Thorac Cardiovasc Surg. 2004;127: 1688-96.

12. Aggarwal NK, Das SN, Sharma G, Kiran U. Efficacy of combined modified and conventional ultrafiltration during cardiac surgery in children. Ann Card Anaesth. 2007; 10:27-33.

13. Davies MJ, Nguyen K, Gaynor JW, Elliott MJ. Modified ultrafiltration improves left ventricular systolic function in infants after cardiopulmonary bypass. J Thorac Cardiovasc Surg. 1998;115:361-9.
14. Chaturvedi RR, Shore DF, White PA, Scallan MH, Gothard JW, Redington AN et al. Modified ultrafiltration improves global left ventricular systolic function after open-heart surgery in infants and children. Eur J Cardiothorac Surg. 1999;15: 742-6.

15. Hodges UM, Berg S, Naik SK, Bower S, Lloyd-Thomas A, Elliot M. Filtration of fentanyl is not the cause of the elevation of arterial blood pressure associated with post-bypass ultrafiltration in children. J Cardiothorac Vasc Anesth. 1994;8: 653-7.

16. Kern FH, Morana NJ, Sears JJ, Hickey PR. Coagulation defects in neonates during cardiopulmonary bypass. Ann Thorac Surg. 1992;54:541-6.

17. Ootaki Y, Yamaguchi M, Oshima Y, Yoshimura N, Oka S. Effects of modified ultrafiltration on coagulation factors in pediatric cardiac surgery. Surg Today. 2002; 32:203-6.

18. Friesen RH, Campbell DN, Clarke DR, Tornabene MA. Modified ultrafiltration attenuates dilutional coagulopathy in pediatric open heart operations. Ann Thorac Surg. 1997;64:1787-9.

19. McEwan A. Aspects of bleeding after cardiac surgery in children. Paediatr Anaesth. 2007;17:1126-33.

20. Keenan HT, Thiagarajan R, Stephens KE, Williams G, Ramamoorthy C, Lupinetti FM. Pulmonary function after modified venovenous ultrafiltration in infants: a prospective, randomized trial. J Thorac Cardiovasc Surg. 2000;119: 501-5.

21. Onoe M, Oku H, Kitayama H, Matsumoto T, Kaneda T. Modified ultrafiltration may improve postoperative pulmonary function in children with a ventricular septal defect. Surg Today. 2001;31:586-90.

22. Allan CK, Newburger JW, McGrath E, Elder J, Psoinos C, Laussen PC, et al. The relationship between inflammatory activation and clinical outcome after infant cardiopulmonary bypass. Anesth Analg. 2010;111:1244-51.

23. Wang W, Huang HM, Zhu DM, Chen H, Su ZK, Ding WX. Modified ultrafiltration in paediatric cardiopulmonary bypass. Perfusion. 1998;13:304-10.

24. Journois D, Israel-Biet D, Pouard P, Rolland B, Silvester W, Vouhé P, et al High-volume, zero-balanced hemofiltration to reduce delayed inflammatory response to cardiopulmonary bypass in children. Anesthesiology. 1996;85 965-76.

25. Jüni P, Holenstein F, Sterne J, Bartlett C, Egger M. Direction and impact of language bias in meta-analyses of controlled trials: empirical study. Int J Epidemiol. 2002;31:115-23 